\title{
A Large and Homogeneous Sample of CMDs of LMC Stellar Clusters
}

\author{
Enzo Brocato \\ Osservatorio Astronomico di Teramo, Via Maggini, I-64100 Teramo, \\ Italy, (email : brocato@astrte.te.astro.it) \\ Elisa Di Carlo \\ Osservatorio Astronomico di Teramo, Via Maggini, I-64100 Teramo, \\ Italy, (email : dicarlo@astrte.te.astro.it)
}

\begin{abstract}
.
We present the photometric results of 21 stellar clusters of the Large Magellanic Cloud. The WFPC2 images were retrieved from the HST archive. Simple stellar populations in a large spread of age are well represented in the sample of color-magnitude diagrams shown here.
\end{abstract}

\section{Introduction and data reduction}

LMC stellar clusters represent an appealing opportunity of probing our understanding of stellar populations in a wide range of age. Clearly, one of the most powerful tools for this purpose is to study their CMDs. The HST collected a number of images all along the years and we decided to use the archive facility to derive a set of homogeneous CMDs for the LMC clusters.

All the images were retrieved from the HST archive as observed with the WFPC2 camera in the filters F450W and F555W. DAOPHOT II (Stetson 1987) was used to obtain the instrumental magnitudes. We followed Dolphin (2000) to derive the calibrated magnitudes and colors.

We find good agreement (within $\simeq 0.1 \mathrm{mag}$ ) between our data and the results of Sarajedini (1998) for the common clusters NGC 2155, SL 663, and NGC 2121. We performed an additional check of our photometry by comparing our data of NGC 2257 to the ground based photometry by Walker (1989). Again the agreement turned out to be very satisfactory (within $\simeq 0.05 \mathrm{mag}$ ).

\section{Results and Conclusions}

After the calibration procedure we derived the CMDs. The 20 younger clusters are shown in Fig.1. The photometric data of all the stars measured in the HST frames $(\mathrm{PC}+\mathrm{WFs})$ are presented without applying any selection.

A deep discussion of the derived CMD of each cluster is beyond the aim of this poster. Thus we present a table (Tab. 1), in which we summarize the main observational features (see Brocato, Di Carlo \& Menna 2001 for more details). 
Table 1. Selected quantities for the LMC clusters of the studied sample. Evaluations with large uncertainties are marked by colon.

\begin{tabular}{cccccc}
\hline Cluster & SWB class & s-parameter & $V_{T O}$ & $V_{H e}$ & $R_{c}(\operatorname{arcsec})$ \\
\hline NGC 2004 & I & 15 & $14.6:$ & $13.8:$ & 9.0 \\
NGC 2100 & I & 17 & $15.2:$ & $13.8:$ & 12.5 \\
NGC 1847 & I & 21 & $15.9:$ & $14.8:$ & 13.8 \\
NGC 2136 & III & 26 & 16.9 & $15.5:$ & 12.0 \\
NGC 1856 & IVA & 30 & 17.8 & 17.75 & 9.0 \\
SL 747 & IVA & - & 18.2 & $17.2:$ & 19.3 \\
NGC 1831 & IVA & 31 & $18.3:$ & 18.45 & 17.5 \\
NGC 2249 & IVB & 34 & 19.0 & 18.7 & 11.5 \\
NGC 1868 & IVA & 33 & 19.2 & 19.0 & 11.0 \\
NGC 2162 & V & 39 & 19.5 & 19.15 & 16.1 \\
NGC 1777 & IVB & 38 & 19.6 & 19.45 & 16.0 \\
NGC 2209 & IVB & 35 & 19.7 & 19.35 & 22.5 \\
NGC 2213 & V & 39 & 19.9 & 19.15 & 12.0 \\
NGC 2173 & VI & 42 & 20.0 & 19.15 & 12.5 \\
NGC 1651 & V & 39 & 20.3 & 19.25 & 17.5 \\
NGC 1718 & VI & 45 & 20.5 & 19.55 & 9.5 \\
SL 506 & V & 46 & 20.5 & 19.25 & 13.3 \\
NGC 2155 & VI & 45 & 20.6 & 18.85 & 16.1 \\
SL 663 & - & 36 & 20.7 & 19.05 & 20.7 \\
NGC 2121 & VI & 44 & $20.8:$ & 19.05 & 29.9 \\
NGC 2257 & VII & 51 & 22.4 & 19.05 & 35.5 \\
\hline \hline
\end{tabular}

We note that very similar observational conditions, the resolving power of the HST and the uniformity of the reduction procedures allow us to produce a homogeneous sample of CMDs of a set of simple stellar populations of very different ages. The present sample can be enlarged to improve the completeness of the CMDs morphology but it is also a powerful database to investigate the evolution of the LMC stellar cluster system. Finally, thanks to the large number of stars measured in each cluster, this sample is also ideal to probe the predictions of stellar evolutionary models in a wide range of masses $(0.8 \mathrm{Mo}-20 \mathrm{Mo})$. This project will proceed by improving the number of CMDs available for the LMC stellar clusters system but also by extending the work to the SMC.

\section{References}

Brocato, E., Di Carlo, E. \& Menna, G. 2001, A\&A, 374, 523

Dolphin, A. E. 2000, PASP, 112, 139

Sarajedini, A. 1998, AJ, 116,738

Walker, A. R. 1989, AJ, 98, 2086 


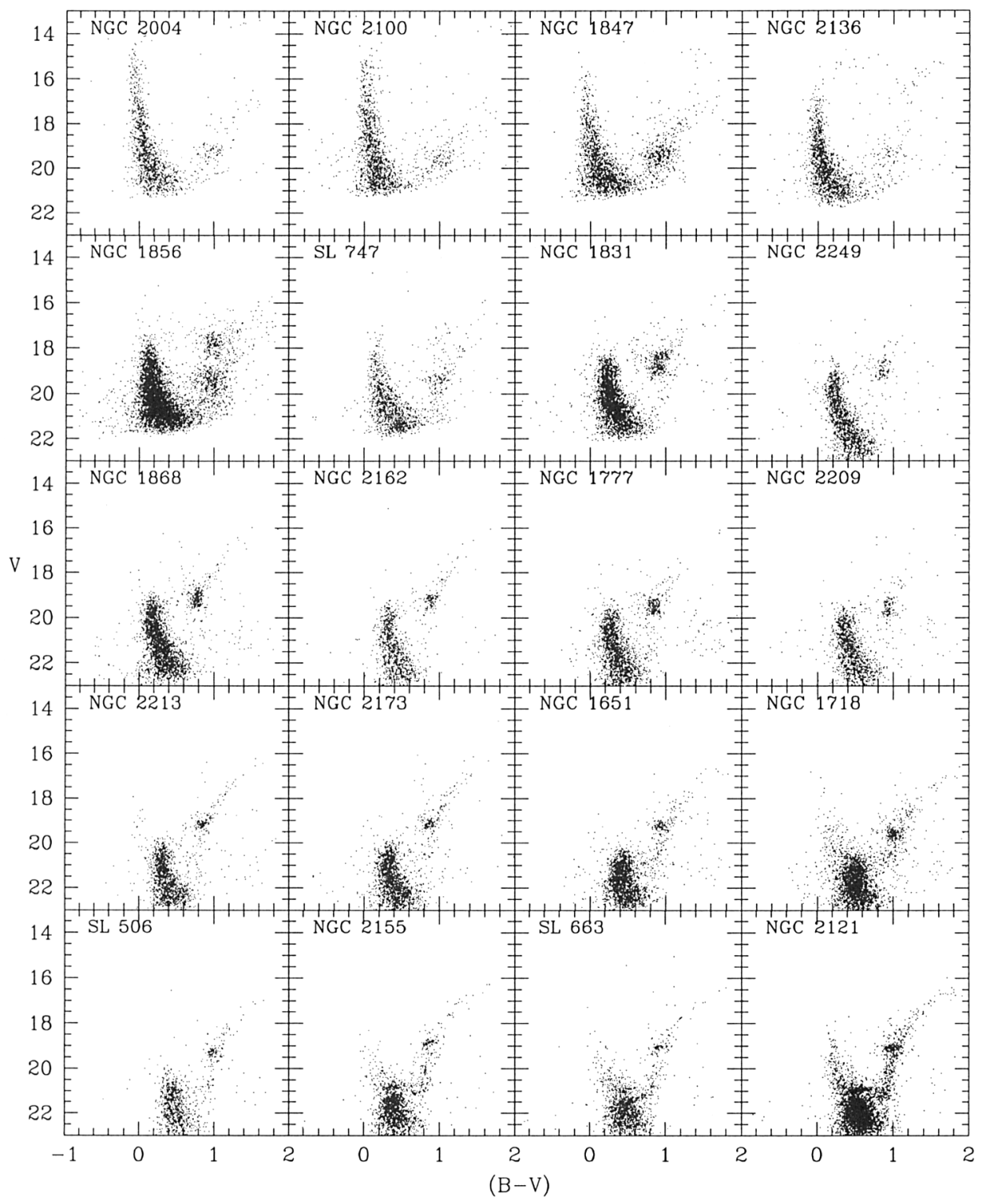

Figure 1. Color-magnitude diagrams for $20 \mathrm{LMC}$ clusters derived in the present work. 